\title{
Intruder Detection Performance of SIMO and MIMO Sensors with Same Number of Channel Responses
}

\author{
Keita USHIKI $^{\dagger}$, Student Member, Kentaro NISHIMORI ${ }^{\dagger a)}$, Senior Member, Naoki HONMA ${ }^{\dagger \dagger}$, \\ and Hideo MAKINO ${ }^{\dagger \dagger}$, Members
}

\begin{abstract}
SUMMARY Intruder detection method by utilizing a time variation of Multiple Input Multiple Output (MIMO) channel (MIMO Sensor) has been proposed. Although the channel capacity on the MIMO transmission is severely degraded in time variant channels, we can take advantage of this feature in MIMO Sensor applications. We have already demonstrated the effectiveness of $2 \times 2$ MIMO sensor using $2.4 \mathrm{GHz}$ band at a small room (Size is $50 \mathrm{~m}^{2}$ ). In this paper, we compare the detection probability of SIMO/MIMO sensors when the number of channel responses are same between SIMO/MIMO sensors: The numbers of transmit and receive antennas are 1 and 4 (SIMO), it is clarified that 2 and 2 (MIMO). The measurement was carried out at the room with the size of $140 \mathrm{~m}^{2}$. From the measured results, $2 \times 2 \mathrm{MIMO}$ sensor obtains the same or higher detection probability compared to $4 \times 1$ SIMO sensor regardless of the measured location.

key words: MIMO Sensor, SISO sensor, time variant channel, channel matrix
\end{abstract}

\section{Introduction}

Reliable security systems have been recently attracted much attention. Radiowave sensors using existing signals, such as the radio using Frequency Modulation (FM), Television (TV) broadcast signals and so on, have been studied, because the radiowave sensors can detect the signal even in Non Line of Sight (NLoS) environment unlike conventional infrared light and camera for security usage.

The radiowave sensors by using UWB-IR and received signal strength indicator (RSSI) based detection method etc. are proposed [1], [2]. The former method needs delay profile measurement and wideband signals are required, hence, the system becomes very complex. The latter method is relatively simple but there is a issue for the detection accuracy [3]. Moreover, since VHF-FM and UHF-TV signals are used in [2], the variation of the propagation channel from the outdoor to indoor might be occurred due to the change in outdoor propagation environment. In order to solve these problems, an intruder detection using array signal processing is proposed [3], [4]. In this method, Single Input Multiple Output (SIMO) channel is assumed and the variation of 1 st eigenvector, which is obtained by the correlation matrix of the received signal, is utilized as a cost function of

\footnotetext{
Manuscript received January 21, 2013.

Manuscript revised May 19, 2013.

$\dagger$ The authors are with the Faculty of Engineering, Niigata University, Niigata-shi, 950-2102 Japan.

${ }^{\dagger}$ The authors are with the Faculty of Engineering, Iwate University, Morioka-shi, 020-8551 Japan.

a)E-mail: nishimori@m.ieice.org

DOI: 10.1587/transcom.E96.B.2499
}

intruder detection.

We proposed an intruder detection method which utilizes channel matrix in Multiple Input Multiple Output (MIMO) channels [5], in order to enhance detection performance in [3]. We call this method MIMO Sensor [6]. Although the channel capacity on the MIMO transmission is severely degraded in time variant channels [7], we can take advantage of this feature in MIMO Sensor applications. Since not only receiving but also transmitting diversity effects are obtained by using MIMO transmission, higher reliability for the intruder detection is expected by using the MIMO Sensor compared to the SIMO sensor. We proposed an use of time correlation function for channel matrix in the MIMO channel [6]. The time correlation using the channel matrix has been compared with that using eignevectors, when 2x2 MIMO system is considered [8]. It is shown that the detection performance by using the channel matrix is almost same with that by the eigenvectors [8]. Since the calculation complexity using the channel matrix is obviously small compared to that using the eigenvector, we adopt the use of time correlation with the channel matrix in MIMO channel in this paper.

In this paper, we evaluate the following topics to clarify the further possibility of SIMO/MIMO sensors when considering the distributed antenna configuration.

- $4 \times 2$ MIMO-OFDM testbed, which has four receivers and two transmitters, is developed. We focus on the detection performance of SIMO/MIMO sensors which have same number of channel responses: $4 \times 1$ SIMO and $2 \times 2$ MIMO sensors are compared.

- The detection performance by not only $2.4 \mathrm{GHz}$ band but also $400 \mathrm{MHz}$ band is clarified, to show the possibility of lower frequency band.

- The detection performance is evaluated at a large room (size is $140 \mathrm{~m}^{2}$ ), to clarify the further possibility of SIMO/MIMO sensors.

The rest of the paper is organized as follows. In Sect. 2, the proposed method, which utilizes the variation on the channel matrix in MIMO channel, is shown. Sect. 3 describes the detailed measurement environment and MIMOOFDM testbed. In Sect. 4, we compare the performance by SIMO and MIMO sensors when considering a large room (size is $140 \mathrm{~m}^{2}$ ). Finally, Sect. 5 concludes the paper. 


\section{Principle of MIMO Sensor}

Figure 1 shows a concept of MIMO sensor [6]. Figures 1(a) and (b) compare the variation of channel matrix in the MIMO channel due to a person. Although the channel capacity of MIMO transmission is severely degraded in the time variant channels [7], we utilize the variation of channel matrix in MIMO channel as an input of Sensor. When $M$ and $N$ are the numbers of transmitting and receiving antennas, the channel matrix $\boldsymbol{H} \in \mathbb{C}^{N \times M}$ is change to $\boldsymbol{H}^{\prime} \in \mathbb{C}^{N \times M}$ due to the intrusion by the person. We realize the intrusion detection by checking the variation of the channel matrix, $\boldsymbol{H}$. The variation of the channel matrix can be expressed by time correlation function. Let us assume that $h_{n o, i j}(i=1 \sim N, j=1 \sim M)$ is a component at the channel matrix without people in the room. When $h_{i j}(t)$ is a component of the channel matrix at time $t$, the time correlation, $\rho_{H}(t)$ is represented by

$$
\rho_{\mathrm{H}}(t)=\frac{\left|\sum_{i=1}^{N} \sum_{j=1}^{M} h_{n o, i j}^{*} h_{i j}(t)\right|}{\sqrt{\sum_{i=1}^{N} \sum_{j=1}^{M}\left|h_{n o, i j}\right|^{2}} \sqrt{\sum_{i=1}^{N} \sum_{j=1}^{M}\left|h_{i j}(t)\right|^{2}}} .
$$

When using SIMO sensor, the number of transmit antenna, $M$ is set to be 1 in Eq. (1). We have to judge whether the intrusion is employed or not by using the value which is obtained in Eq. (1). There are two reasons why Eq. (1) becomes small.

(a) Variation on channel matrix due to the intrusion or surrounding environment

(b) Variation on channel matrix due to thermal noise

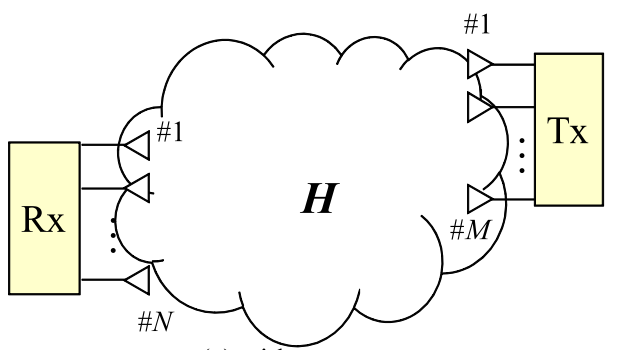

(a) without person $\quad \# 1$



(b) with person

Fig. 1 Concept of MIMO sensor (Tx: Transmitter, Rx: Receiver).
We evaluate influence on the time correlation by the thermal noise, because the influence by only reason (a) must be detected. The correlation considering the thermal noise $\rho_{H, w / n}$ is represented as

$$
\begin{aligned}
\boldsymbol{H}_{w / n}= & \boldsymbol{H}_{w / o, n}+\frac{1}{\operatorname{SNR}} \boldsymbol{H}_{n}, \\
\rho_{H, w / n}= & \mid \frac{\left|\sum_{i=1}^{N} \sum_{j=1}^{M} h_{w / o, n, i j}^{*} h_{w / n, i j}\right|}{\sqrt{\sum_{i=1}^{N} \sum_{j=1}^{M}\left|h_{w / o, n, i j}\right|^{2}} \sqrt{\sum_{i=1}^{N} \sum_{j=1}^{M}\left|h_{w / n, i j}\right|^{2}}},
\end{aligned}
$$

where $\boldsymbol{H}_{w / n} \in \mathbb{C}^{N \times M}$ and $\boldsymbol{H}_{w / o, n} \in \mathbb{C}^{N \times M}$ are the channel matrix with/without the thermal noise, respectively. $\boldsymbol{H}_{n} \in$ $\mathbb{C}^{N \times M}$ is the matrix by the thermal noise. $h_{w / n, i j}$ and $h_{w / o n, i j}$ $(i=1 \sim N, \quad j=1 \sim M)$ are components on the channel matrix.

Since it is difficult to obtain the ideal channel matrix without the thermal noise, the channel matrix is estimated with the sufficient number of samples when considering the static channel without the person inside the room in advance. We regard this estimated channel matrix $\boldsymbol{H}_{w / o, n}$. After the estimation of $\boldsymbol{H}_{w / o, n}$, we artificially add the thermal noise in order to evaluate the performance by the proposed method versus the SNR. In the measurement, we confirmed that actual SNR for $\boldsymbol{H}_{w / o, n}$ is greater than $30 \mathrm{~dB}$ and this channel matrix is regarded as the ideal channel matrix.

Actual calculation method using Eqs. (1) (3) is explained as follows.

(a) First,the calculation of Eq. (1) is employed, and the SNR is obtained by using the channel matrix, $\boldsymbol{H}$.

(b) $\rho_{H, w / n}$ is calculated by using Eqs. (2) and (3) and SNR which is obtained in Step (a). Since there is Gaussian distribution regarding each component of $\boldsymbol{H}_{n}$, there is a distribution on $\rho_{H, w / n}$. The values of $\boldsymbol{H}_{n}$ can be calculated in advance.

(c) $\rho_{H, w / n}$ is calculated for each $\boldsymbol{H}_{n}$, and $x \%$ value of cumulative distribution function $(\mathrm{CDF}), \rho_{H, w / n}(x \%, \mathrm{SNR})$ is obtained.

(d) The person is judged to be detected when the equation is satisfied as

$$
\rho_{M I M O}<\rho_{H, w / n}(x \%, S N R) .
$$

In this paper, $\rho_{M I M O}$ by Eq. (1) is calculated when the person exists inside the various locations of the room, and the probability where Eq. (4) is satisfied among all the trials is defined the detection probability. In order to exam how the proposed method detects the person inside the room, we evaluate how the person is detected by using Eqs. (1) (4) instead of the intruder detection. Even if Eq. (4) is satisfied, the false alarm may occur with the probability of $x \%$ due to the effect on the thermal noise. We define this probability as false detection rate. In this paper, $x$ is set to be $1 \%$ as one of examples.

In order to obtain the threshold value, $\rho_{H, w / n}(x \%$, 


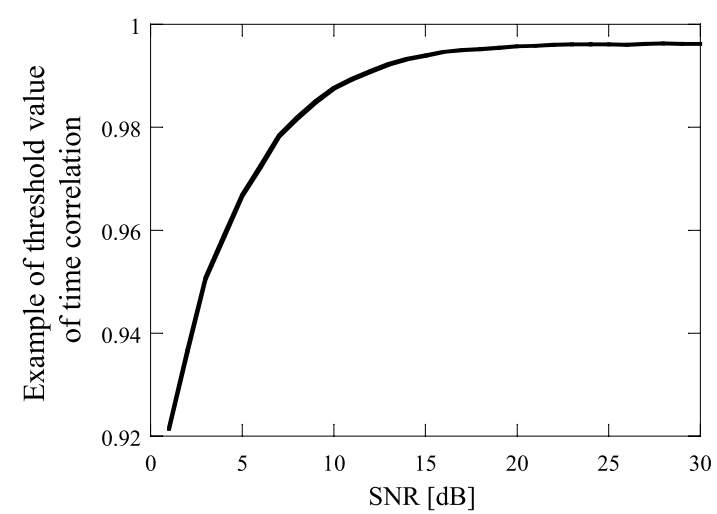

Fig. 2 Example of threshold value of time correlation versus SNR when considering only thermal noise.

SNR), $\boldsymbol{H}_{w / o, n}$ and $\boldsymbol{H}_{n}$ are required as shown in Eqs. (2) and (3). When considering the channel matrix without the person in the room, we confirmed that $\boldsymbol{H}_{w / o, n}$ is not changed [9]. Hence, $\boldsymbol{H}_{w / o, n}$ can be prepared before the calculation in Eq. (1). Moreover, $\boldsymbol{H}_{n}$ can be prepared by using the random values with Gaussian distribution. Hence, threshold value can be prepared in advance before calculating Eq. (4).

Figure 2 shows an example of threshold value of time correlation versus SNR when considering only thermal noise. The false detection probability is $1 \%$. As can be seen in Fig. 2, the correlation is reduced due to thermal noise when the SNR is very small. On the other hand, the correlation is greater than 0.99 when considering $\mathrm{SNR} \geq 12 \mathrm{~dB}$. We calculate this curve for each measurement and use this curve as the threshold for the intruder detection.

\section{Measurement Environment}

To compare the SIMO/MIMO sensors with same number of channel responses, we conducted the measurement in an actual indoor environment. The measurement environment is shown in Fig. 3. The size of room is $8.9 \times 15.8 \times 2.6 \mathrm{~m}$ $\left(140 \mathrm{~m}^{2}\right)$ in $\mathrm{X}, \mathrm{Y}$ and $\mathrm{Z}$ planes. This room is almost triple size of that in evaluated [6]: SIMO/MIMO sensors are compared at such a large room. During the measurement, the transmitter and receiver are fixed at Line-of-sight (LOS) position. The number of transmit and receive antennas are two and four, respectively: $4 \times 2$ MIMO channel measurement can be realized. In this paper, we focus on the detection performance of SIMO/MIMO sensors which have same number of channel responses: SIMO with $(M, N)=(1,4)$ and MIMO with $(M, N)=(2,2)$. Table 1 shows the selected antennas for SIMO and MIMO sensors. Although there is four antennas at the receiver, antennas \#1 and \#4 are selected for the evaluation on the distributed array.

The print dipole antenna, which is known as omnidirectional antenna in the horizontal plane, is used for the transmit and receive antennas [10]. As shown in Fig. 3, the MIMO channels are measured when a person moves on Course A, B, C, D, E and F. The total measurement time were 12 seconds for courses A to D and 8 seconds for

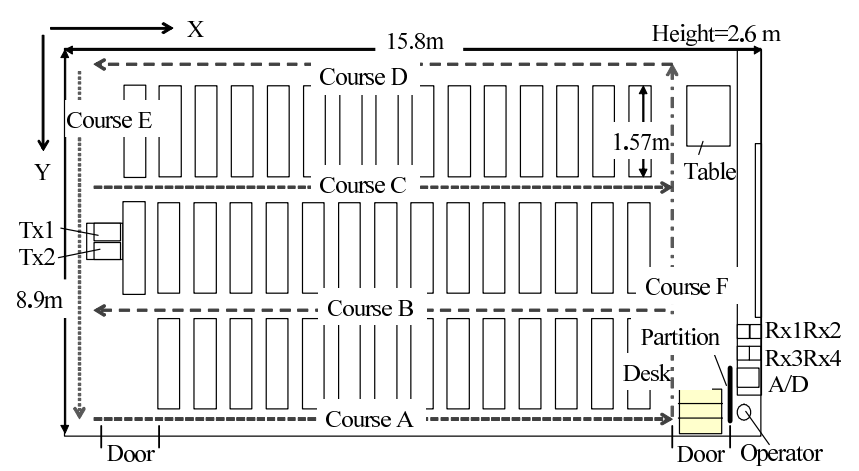

Fig. 3 Measurement environment.

Table 1 Selected antennas.

\begin{tabular}{|c|c|c|c|c|c|c|}
\hline & $\mathrm{Tx} 1$ & $\mathrm{Tx} 2$ & $\mathrm{Rx} 1$ & $\mathrm{Rx} 2$ & $\mathrm{Rx} 3$ & $\mathrm{Rx} 4$ \\
\hline \hline MIMO & $\circ$ & $\circ$ & $\circ$ & & & $\circ$ \\
SIMO (1) & $\circ$ & & $\circ$ & $\circ$ & $\circ$ & $\circ$ \\
SIMO (2) & & $\circ$ & $\circ$ & $\circ$ & $\circ$ & $\circ$ \\
\hline
\end{tabular}

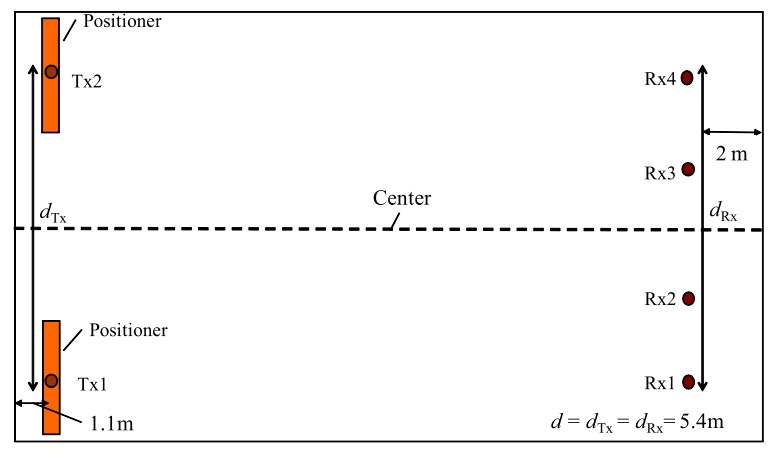

Fig. 4 Antenna arrangement.

courses $\mathrm{E}$ and $\mathrm{F}$, respectively. The number of human subject is two and their body height is approximately $170 \mathrm{~cm}$. The antenna positions for Tx and $\mathrm{Rx}$ in this measurement are shown in Fig. 4. The array width $d_{\mathrm{Tx}}$ and $d_{\mathrm{Rx}}$ at the transmitter and receiver sites are set to be $5.4 \mathrm{~m}$, for the evaluation on the distributed array configuration. $d=d_{\mathrm{Tx}}=d_{\mathrm{Rx}}$. The positions of transmit antennas were moved 10 times with the interval of $6.25 \mathrm{~cm}$ by using a position controller. So as to keep $d=5.4 \mathrm{~m}$. The antenna height at Tx and Rx sites are set to be $1.0 \mathrm{~m}$.

In this paper, the detection performance is also compared due to the difference of the frequency band. We selected not only $2.4 \mathrm{GHz}$ band [6] but also $400 \mathrm{MHz}$ band, because $400 \mathrm{MHz}$ band might be used in RF-ID or sensor systems. Moreover, there is a possibility to utilize high SNR by $400 \mathrm{MHz}$ band. On the other hand, the antenna size by $400 \mathrm{MHz}$ band is larger than that by $2.4 \mathrm{GHz}$ band. In order to fairly compare the performance by these frequency band $(2.4 \mathrm{GHz} / 400 \mathrm{MHz})$, the antenna width in Fig. 4 is fixed to be $5.4 \mathrm{~m}$.

Figure 5 shows MIMO transceiver which is used in this measurement. The bandwidth is $6.25 \mathrm{MHz}$, respectively. The transmit power was changed according to the measure- 
ment. The signal format at the transmitter is shown in Fig. 6 . We adopt OFDM transmission which is incorporated in WLAN systems [11], because W-LAN is widely incorporated in indoor environment and W-LAN signals might be used not only for the communication but also the intruder detection by the proposed method. The main parameters in transceiver and OFDM transmission are denoted in Table 2. The timing synchronization is employed by using the short preamble (SP in Fig. 6). We obtained the channel matrix by using long preamble (LP in Fig. 6) of OFDM signals with the interval of $0.152 \mathrm{~ms}$. The correlation value is obtained in each subcarriers of OFDM signals by using Eqs. (1) and (3).

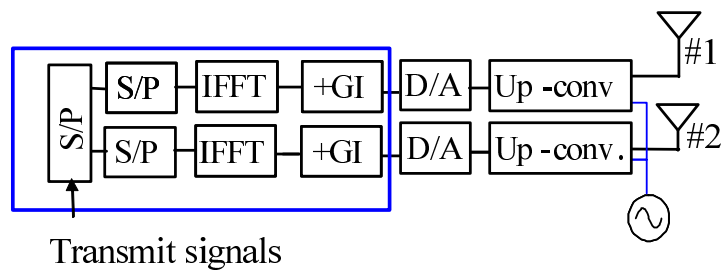

(a) Transmitter

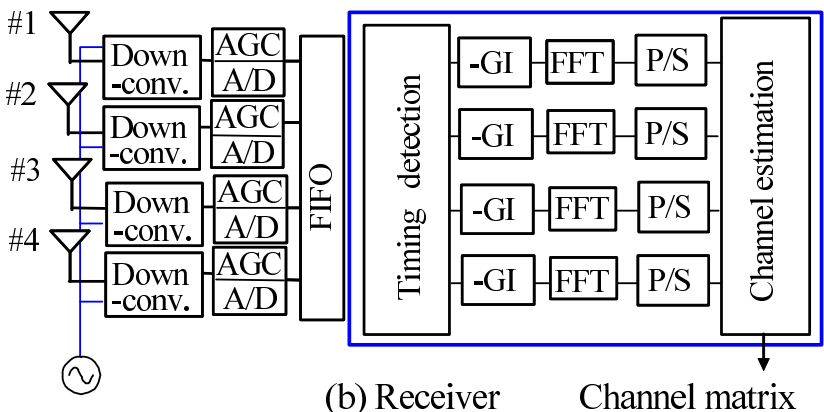

Fig. 5 Transceiver for SIMO and MIMO sensors

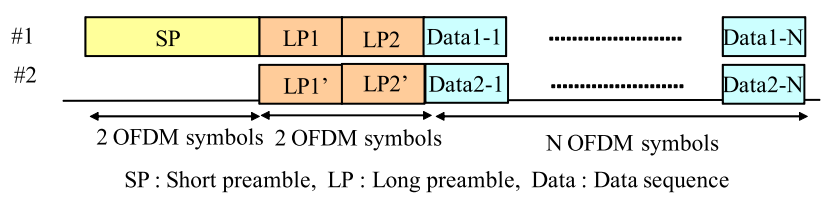

Fig. 6 Signal format at transmitter.

Table 2 Parameters in transceiver and OFDM transmission.

\begin{tabular}{|c|c|}
\hline Frequency & $2.4 \mathrm{GHz} / 400 \mathrm{MHz}$ \\
\hline Bandwidth & $6.25 \mathrm{MHz}$ \\
\hline Sampling rate (A/D) & $25 \mathrm{MHz}$ \\
\hline Sampling rate (D/A) & $25 \mathrm{MHz}$ \\
\hline Number of over-samples & 4 \\
\hline FFT points & 64 \\
\hline Number of sub-carriers & 56 \\
\hline Number of pilot sub-carriers & 4 \\
\hline Number of OFDM symbols & 80 symbol \\
\hline Length of short preamble & 2 OFDM symbols \\
\hline Length of long preamble & 2 OFDM symbols \\
\hline Positions of pilot subcarrier & $6,20,34,48$ \\
\hline
\end{tabular}

\section{Intruder Detection by SIMO/MIMO Sensors}

\subsection{Correlation Characteristics with/without People}

In this subsection, basic performance of $2 \times 2$ MIMO sensor is clarified. Hera, the measured frequency is $440 \mathrm{MHz}$. In order to confirm reliability of this measurement, the time variation of the channel matrix without people in the room is plotted in Fig. 7. Although there is not the person inside the room, there is the person at the corridor near the room in Fig. 7. As can be seen in this figure, since time correlation is greater than 0.9995 (equivalent SNR can be regarded greater than $33 \mathrm{~dB}$ ), we can judge that the propagation environment can be regarded static when considering without the intrusion by people. Hence, we evaluate how the channel matrix is changed with and without the person inside the room instead of the intruder detection performance hereafter, because the channel matrix is not changed outside the room even if there is the person very near the room.

Figure 8 shows the CDF versus the time correlation with and without people in the room. Equations (1) and (3) are used to obtain the CDF of the time correlation for with and without people, respectively. The measured results con-

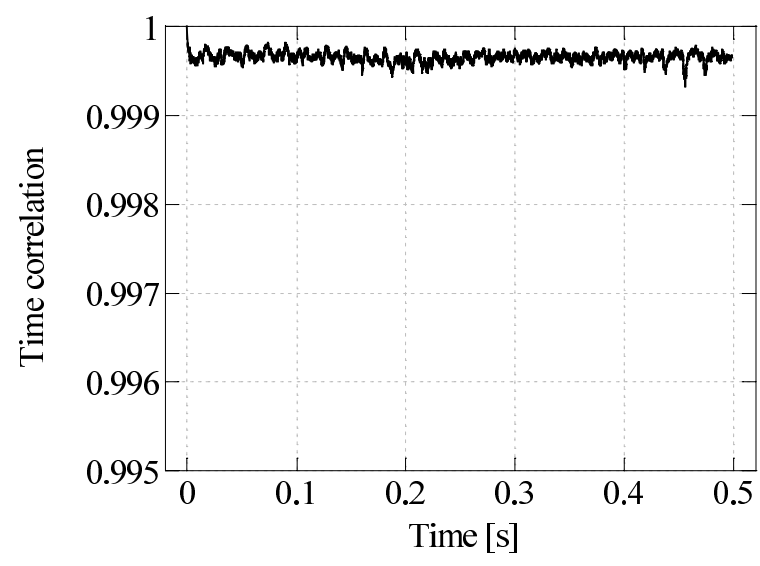

Fig. 7 An example of time correlation without people.

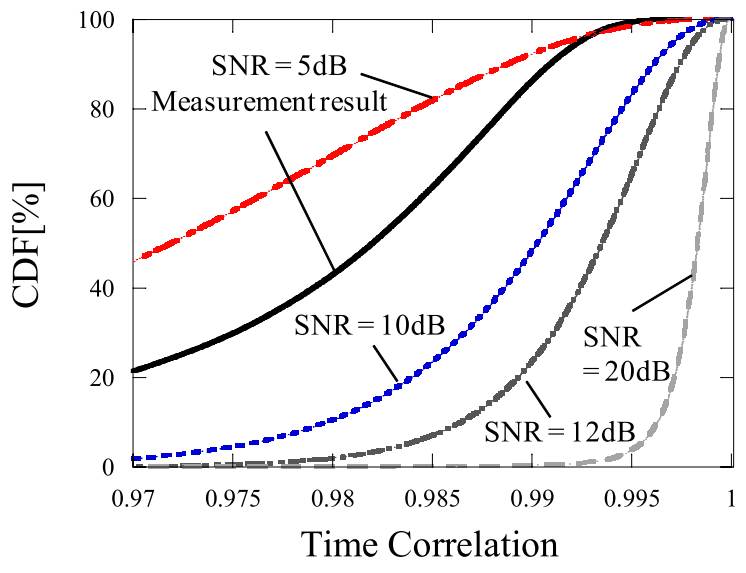

Fig. 8 CDF of time correlation 


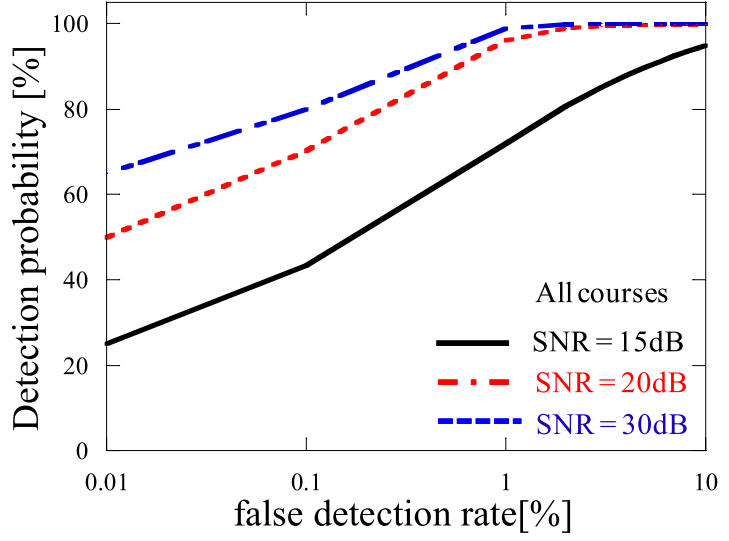

Fig. 9 Detection probability vs. false detection rate (All courses, $\mathrm{SNR}=20 \mathrm{~dB})$.

sidering all the courses are reflected in Fig. 8. Correlations due to the thermal noise are obtained changing the SNR in Eq. (3). As can be seen in Fig. 8, the correlation with people is smaller than that without people with a high probability when the SNR is greater than $10 \mathrm{~dB}$. Hence, the relationship between the detection probability and SNR can be obtained, when the false detection rate is determined.

Figure 9 represents the detection probability versus false detection rate. False detection rate is changed from 0.01 to $10 \%$. The results are plotted in this figure when the SNR is 15, 20 and $30 \mathrm{~dB}$, respectively. As can be seen Fig. 9, the detection probability is changed according to the value of false detection rate, especially, when $\mathrm{SNR}=15 \mathrm{~dB}$. The threshold value as the false detection rate in this paper is set to be $1 \%$ for remaining results.

\subsection{Detection Performance between $2.4 \mathrm{GHz}$ and $400 \mathrm{MHz}$ Bands}

In this subsection, the detection performance by the difference of radio frequency band is evaluated. Figure 10 denotes the detection probability versus the SNR when $2.4 \mathrm{GHz}$ and $440 \mathrm{MHz}$ bands are used, respectively. The results with $d=5.4 \mathrm{~m}$ are plotted in this figure. As can be seen in this figure, the much higher detection performance by using $440 \mathrm{MHz}$ band is obtained compared to $2.4 \mathrm{GHz}$ band. The SNRs which can obtain detection $99 \%$ probability are -19 and $0 \mathrm{dBm}$ at $440 \mathrm{MHz}$ and $2.4 \mathrm{GHz}$ bands, respectively. Since the difference of free space path loss between $440 \mathrm{MHz}$ and $2.4 \mathrm{GHz}$ is approximately $16 \mathrm{~dB}$, the higher detection performance by $400 \mathrm{MHz}$ band is obtained thanks to a lower path loss. Hence, the higher detection performance is expected by lower frequency band due to the lower path loss. Hereafter, we use the antennas whose frequency band is $440 \mathrm{MHz}$ band.

\subsection{Performance Comparison between SIMO and MIMO Sensors}

Figures 11, 12 and 13 compare the detection probabilities

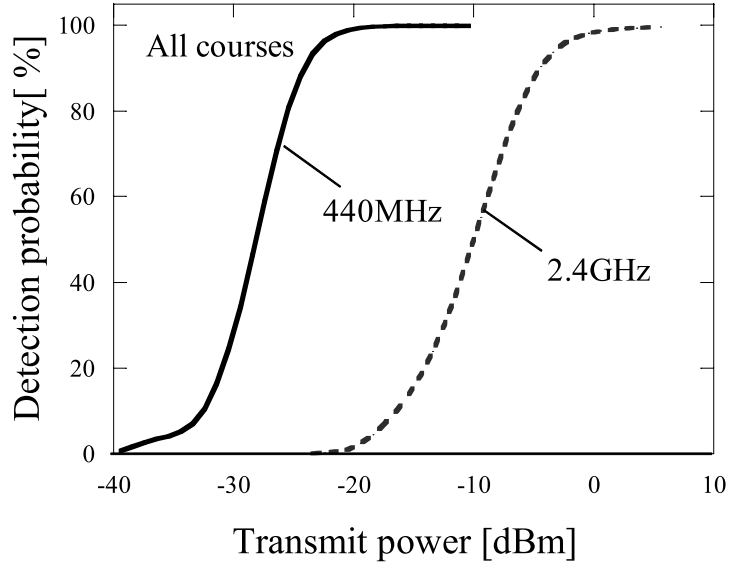

Fig. 10 Detection probability comparison due to the difference of radio frequency.

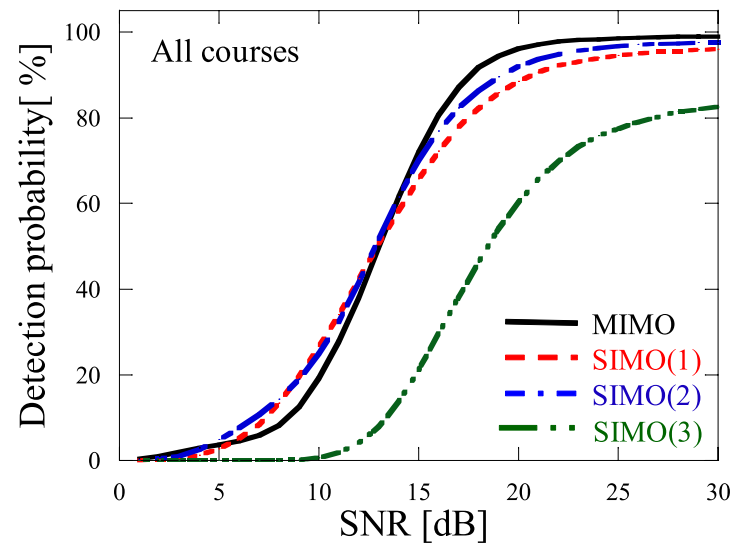

Fig. 11 Detection probability vs. SNR (All courses).

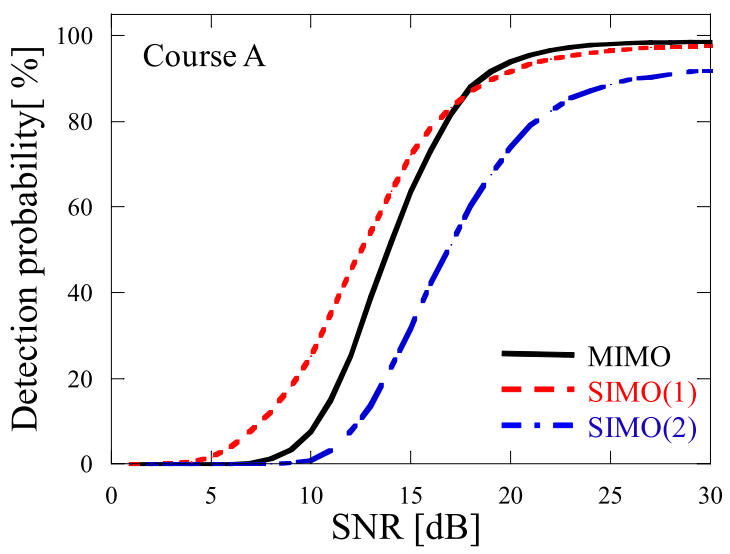

Fig. 12 Detection probability vs. SNR (Course A).

when considering SIMO $(M=1, N=4)$ and MIMO $(M=$ $2, N=2$ ) sensors. The results with all courses, course A and course D are plotted in Figs. 11, 12, and 13, respectively. The noise power is given so that the average SNR is $30 \mathrm{~dB}$ when the transmit power is $-13 \mathrm{dBm}$ probability at course A. Hence, the difference in received power due to antenna width, measured course and antenna height can be evalu- 




Fig. 13 Detection probability vs. SNR (Course D).

ated. Note that the difference among MIMO, SIMO (1), and SIMO (2) sensors can be referred in Table 1. As can be seen in Fig. 11, the detection performance by MIMO is slightly higher than that by SIMO (1) and SIMO (2).

In order to exam the detection performance when considering more realistic antenna arrangement, we evaluated the detection performance when the transmit antenna is located at the center on Y-axis of the room in Fig. 3 and its antenna is also located on the ceiling. The receivers' setting is the same with SIMO (1) and SIMO (2). We call this arrangement SIMO (3). The result is also plotted in Fig. 11. As can be seen in Fig. 11, the detection rate with SIMO (3) is much smaller than the other arrangements and the detection rate is $81 \%$ even if $\mathrm{SNR}=30 \mathrm{~dB}$. In the previous work [6], we have already confirmed that the detection performance is severely degraded when the antenna height is higher than body height. We confirmed that the antenna height strongly affects the detection performance. Hence, we compare MIMO, SIMO (1), and SIMO (2) hereafter.

On the other hand, as can be seen in Fig. 12, it is shown that the detection performance by SIMO (1) is much higher than that by SIMO (2). We also observe that the detection probability by SIMO (1) is lower than that by SIMO (2), as shown in Fig. 13.

To clarify the reason why the merit of detection performance by SIMO sensor is changed with respect to each course, the detection rate versus the time of walking is plotted in Figs. 14 and 15 for courses A and D. The SNR is $20 \mathrm{~dB}$ and a person moves with about $1 \mathrm{~m}$ per second. The detection probability is calculated with the interval of $1 \mathrm{~m}$. As shown in Fig. 15, over 80\% probability is obtained at almost every time when considering MIMO and SIMO (1) sensors. On the other hand, the detection probability is about $60 \%$ with the time of 0 to 6 second when SIMO (2) sensor is applied, because the transmit antenna \#1 is located at course A and the transmit antenna \#2 is far from course A. When we focus on the results in Fig. 15 for course D, the detection probability by SIMO (1) is smaller than that by MIMO and SIMO (2), because the transmit antenna \#2 is located at course D and the transmit antenna \#1 is far from course D.



Fig. 14 Detection probability vs. measurement time (Course A).

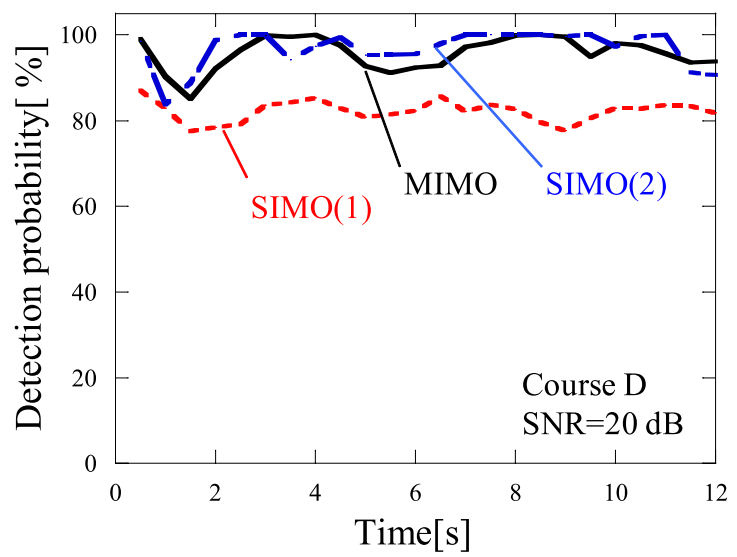

Fig. 15 Detection probability vs. measurement time (Course A).

Table 3 Detection probability due to the antenna composition [\%] (SNR $=20[\mathrm{~dB}]$ ).

\begin{tabular}{|c|r|r|r|}
\hline Course & SIMO(1) & SIMO(2) & MIMO \\
\hline \hline A & 91.6 & 73.9 & 93.9 \\
B & 92.2 & 90.9 & 97.6 \\
C & 80.9 & 99.8 & 97.4 \\
D & 82.2 & 96.8 & 95.8 \\
E & 86.5 & 92.8 & 95.5 \\
F & 97.5 & 97.9 & 96.5 \\
\hline All & 88.5 & 92.0 & 96.1 \\
\hline
\end{tabular}

From these results, we confirmed that the detection probability by the SIMO sensor might be degraded due to single antenna at the transmitter site while the MIMO sensor obtains the high detection probability by both transmit and receive diversity effect: robust performance is expected by the MIMO sensor.

Table 3 shows the detection probability due to the antenna composition when the SNR is $20 \mathrm{~dB}$.

\section{Conclusion}

In this paper, we compared the detection performance of SIMO/MIMO sensors which have same number of channel responses at a large room $\left(140 \mathrm{~m}^{2}\right)$ : $4 \times 1$ SIMO and 
$2 \times 2$ MIMO. It is shown that the detection probability by $4 \times 1$ SIMO sensor might be degraded due to single antenna at the transmitter site while $2 \times 2$ MIMO sensor obtains the high detection probability by both transmit and receive diversity effect. Moreover, the use of $400 \mathrm{MHz}$ band is effective compared to $2.4 \mathrm{GHz}$ band, because the SNR is more important than the spatial correlation. Although $1 \%$ is adopted as the value of false detection rate, lower values should be evaluated as the false detection rate in the future work for more realizable system.

Although we deal with the time correlation using only the channel matrix, there may be a change to improve the detection rate using the eigenvectors when considering the number of transmit and receive antennas are greater than two, because the multiple eigenvectors are utilized. As the future work, the effectiveness of the use on the eigenvectors should be evaluated.

\section{Acknowledgment}

The part of this work is supported by KAKENHI, Grantin-Aid for Scientific Research (C) (25420362) and by the Telecommunication Advancement Foundation in 2013. The authors would like to thank for the members of Nishimori Laboratory for the help on the measurement.

\section{References}

[1] K. Terasaka, K. Higashikaturagi, I. Matunami, and A. Kajiwara, "Human body detection using UWB-IR indoor channel," Proc. IEEE International Symposium on Personal Indoor and Mobile Radio Communication 2007 (PIMRC2007), Sept. 2007.

[2] M. Nishi, S. Takahashi, and T. Yoshida, "Indoor human detection systems using VHF-FM and UHF-TV broadcasting waves," Proc. IEEE International Symposium on Personal Indoor and Mobile Radio Communication 2006 (PIMRC2006), Session TJ7.3, Sept. 2006.

[3] S. Ikeda, H. Tsuji, and T. Ohtsuki, "Indoor event detection with eigenvector spanning signal subspace for home or office security," IEICE Trans. Commun., vol.E92-B, no.7, pp.2406-2412, July 2009

[4] S. Ikeda, T. Ohtsuki, and H. Tsuji, "Signal-subspace-partition event filtering for eigenvector-based security system using radio waves," IEEE International Symposium on Personal Indoor and Mobile Radio Communications (PIMRC2009), Tokyo, Japan, Sept. 2009.

[5] D. Gesbert, et al., "From theory to practice: An overview of MIMO space-time coded wireless systems," IEEE J. Sel. Areas Commun., vol.21, no.3, pp.281-302, April 2003.

[6] K. Nishimori, Y. Koide, D. Kuwahara, N. Honma H. Yamada, and H. Makino, "MIMO sensor - Evaluation on antenna arrangement," Proc. EuCAP2011, pp.2924-2928, April 2011.

[7] J.W. Wallace and M.A. Jensen, "Time-varying MIMO channels: Measurement, analysis, and modeling," IEEE Trans. Antennas Propag., vol.54, no.11, pp.3265-3273, Nov. 2006.

[8] T. Sugiura, N. Honma, and K. Nishimori, "MIMO sensorRaytracing analysis of break-in detection performance in indoor environment,” IEICE Technical Report, AP2010-76, Oct. 2010.

[9] K. Nishimori, R. Kataoka, and H. Makino, "MIMO transceiver for both channel sounding and bitrate measurement," IEICE Technical Report, AMT2012-15, Dec. 2102.

[10] S.R. Saunders, Antenna and Propagation for wireless communication system, John Willy \& Sons, New York, 1999.

[11] IEEE802.11n, http://www.ieee802.org/11/

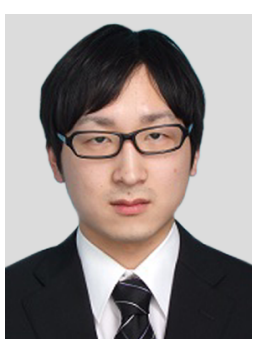

Keita Ushiki received the B.E. degree from faculty of engineering in Niigata University in 2012. He is currently studying M.S. degree at graduate school of science and technology in Niigata University. His research interest is MIMO sensors.



Kentaro Nishimori received the B.E., M.E. and $\mathrm{Ph} . \mathrm{D}$. degrees in electrical and computer engineering form Nagoya Institute of Technology, Nagoya, Japan in 1994, 1996 and 2003, respectively. In 1996, he joined the NTT Wireless Systems Laboratories, Nippon Telegraph and Telephone Corporation (NTT), in Japan. He was senior research engineer on NTT Network Innovation Laboratories. He is now associate professor in Niigata University. He was a visiting researcher at the Center for Teleinfrastructure (CTIF), Aalborg University, Aalborg, Denmark from Feb. 2006 to Jan. 2007. He was an Associate Editor for the Transactions on Communications for the IEICE Communications Society from May 2007 to May 2010 and Assistant Secretary of Technical Committee on Antennas and Propagation of IEICE from June 2008 to May 2010. He received the Young Engineers Award from the IEICE of Japan in 2001, Young Engineer Award from IEEE AP-S Japan Chapter in 2001, Best Paper Award of Software Radio Society in 2007 and Distinguished Service Award from the IEICE Communications Society in 2005, 2008 and 2010. His main interests are spatial signal processing including MIMO systems and interference management techniques in heterogeneous networks. He is a member of IEEE. He received IEICE Best Paper Award in 2010.

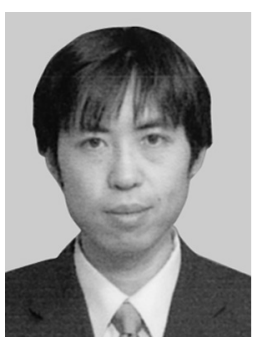

Naoki Honma received the B.E., M.E., and Ph.D. degrees in electrical engineering from Tohoku University, Sendai, Japan in 1996, 1998 , and 2005, respectively. In 1998, he joined the NTT Radio Communication Systems Laboratories, Nippon Telegraph and Telephone Corporation (NTT), in Japan. He is now working for Iwate University. He received the Young Engineers Award from the IEICE of Japan in 2003 , the APMC Best Paper Award in 2003, and the Best Paper Award of IEICE Communication Society in 2006, respectively. His current research interest is planar antennas for high-speed wireless communication systems. He is a member of IEEE.

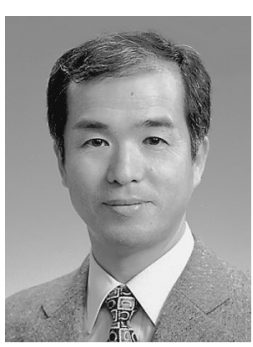

Hideo Makino graduated from the Department of Electronic Engineering, Niigata University, in 1976, completed the M.S. program in 1978, and joined the staff of the Department of Information Engineering. He became an associate professor in 1990 and a professor in 1995. He was on leave at the Research Institute of Applied Electrical Engineering, Hokkaido University, in 1983 and at the Medical School, University of Toronto, in 1989. He has been engaged in research on medical information and assistive equipment. He holds a D. Eng. degree, and is a member of the Japan ME Society, the Institute of Electrical Engineers of Japan, and IEEE. He is a council member of the Japan Heart Rhythm Society and a councilor of the Geographical Information Systems Society. 